\title{
PERSPECTIVE
}

\section{Should diabetic patients be screened for glaucoma?}

\author{
John D Ellis, Andrew D Morris, Caroline J MacEwen for the DARTS/MEMO Collaboration
}

Untreated primary open angle glaucoma (POAG) is a blinding optic neuropathy. Timely medical or surgical treatment is effective in slowing the rate of optic nerve damage and delaying the onset of visually disabling field loss, ${ }^{1}$ but treatment is only effective in prevention, and there is as yet no means of reversing established axon loss. In the United Kingdom, around 250000 people suffer from POAG with visual field loss. ${ }^{23}$ The disease accounts for $15 \%$ of the 1500 new registrations for blindness in England and Wales per year. ${ }^{4}$

The insidious nature of POAG, its potential detection by relatively simple, non-invasive, screening tests at an early stage, and the existence of treatments effective at preventing disabling visual loss constitute a cogent argument for screening. Consistently, over many years and in numerous different populations, researchers have found that $50 \%$ of the disease remains undetected in the community, ${ }^{6}$ and one in five of those with newly confirmed disease already have advanced field loss. ${ }^{7}$ Clearly, new approaches to the detection of POAG are required if we detect only half the prevalent disease. To target individuals at increased risk would appear to constitute sound clinical and economic practice. $^{89}$

The "Guidelines for the management of ocular hypertension and primary open angle glaucoma"10 recently published by the Royal College of Ophthalmologists, define risk factors for the development of POAG. These include a positive family history in a first degree relative, high myopia, black race, and diabetes. These groups would therefore appear suitable for targeted screening.

The purpose of this review is to examine the evidence that one of these factors, diabetes mellitus, is a risk factor for POAG. It is recommended that all people with diabetes should receive annual screening for diabetic retinopathy, ${ }^{11}$ and if there truly is an association between diabetes and POAG, it is tempting to speculate that annual assessment should include a measurement of intraocular pressure (IOP) and that all newly diagnosed glaucoma patients should receive a random blood glucose test.

Related areas of controversy including the protective influence of elevated pressure in the development of diabetic retinopathy ${ }^{12}$ and the suggestion that diabetic patients may have an excess risk of primary angle closure glaucoma ${ }^{13}$ are beyond the scope of this review.

Glaucoma and diabetes: is there an association? The association between diabetes and POAG is not new. In 1971 Becker stated “ . . diabetes mellitus occurs more often in patients with primary open angle glaucoma than in non-glaucomatous populations. Similarly glaucoma is more prevalent in diabetic than in non-diabetic populations." ${ }^{\prime 4}$ This suggested a greater degree of consensus than was warranted at the time, as there was conflicting evidence with some studies reporting an association between diabetes and $\mathrm{POAG}^{15-18}$ and others reporting no association. ${ }^{19-23}$ We have performed a critical review of the evidence to clarify the picture.

We reviewed all potentially relevant English language articles from 1966 to 1997 (further papers obtained from bibliography). Inevitably this approach may have introduced bias by the inadvertent omission of non-English language studies. Additionally, there may be other unpublished studies which intuitively are more likely to be negative. ${ }^{24}$

Available studies vary in three principal factors; (i) the population studied-that is, whether patients were selected from the general population or from hospital clinics; (ii) the criteria used to diagnose diabetes; and, (iii) the criteria used to define and diagnose glaucoma.

All studies which have evaluated the association are shown in Table 1. Most of the earlier studies (before 1971) were small, used differing definitions of glaucoma, and were clinic rather than community based and thus examined a selected population. ${ }^{17} 192022232526$ Those that looked at the prevalence of glaucoma, rather than at intraocular pressure only, generally included intraocular pressure as part of the definition of the disease, making it difficult to separate an association of diabetes with ocular hypertension and to exclude low tension glaucoma.

For example, Armstrong et al ${ }^{17}$ examined 393 unselected diabetic patients attending a hospital clinic and 280 age and sex matched controls from a general medical clinic, and reported a prevalence of POAG of $4.1 \%$ in the diabetic patients compared with $1.4 \%$ in the controls. Definite glaucoma was defined as the presence of an IOP $>26.5 \mathrm{~mm} \mathrm{Hg}$, regardless of field defects. This inevitably included some cases of ocular hypertension. The investigators went on to examine the case records of all subjects who attended the glaucoma clinic $(n=325)$ to ascertain a history of diabetes. This revealed a prevalence of diabetes of $12.6 \%$. However, cases of secondary glaucoma accounted for one third of this group and data on glaucoma type were incomplete. The authors stated that a prevalence of diabetes of $12 \%$ is more than would be expected from a normal population thus supporting an association between diabetes and glaucoma. The average age of the patient attending a glaucoma clinic is likely to be high (not stated by the authors) and population prevalence figures for diabetes in white subjects over 65 years of $8-14.7 \%$ have been reported. ${ }^{27}$ The significance of their findings is therefore uncertain. This study was limited by being clinic based, small, inadequately controlled, and incorporating Schiøtz tonometry pressure as the only diagnostic criterion for glaucoma.

In contrast, the Bedford Eye Survey ${ }^{28}$ studied 3072 people aged over 40 randomly selected from the general population. Ocular examination included IOP measurement, examination of the optic discs, and Lister perimeter fields. Examination for diabetes included urinalysis and an oral glucose tolerance test (OGTT) in all patients with glycosuria $(n=212)$. The overall prevalence of glaucoma 
Table 1 Studies of association between diabetes mellitus, intraocular pressure, and glaucoma

\begin{tabular}{|c|c|c|c|c|c|c|c|c|c|c|}
\hline \multirow[b]{2}{*}{ Author(s) } & \multirow[b]{2}{*}{ Published } & \multirow[b]{2}{*}{ Location } & \multirow[b]{2}{*}{ Method } & \multirow[b]{2}{*}{ Numbers } & \multicolumn{2}{|l|}{ Ascertainment } & \multirow{2}{*}{$\begin{array}{l}\text { Association } \\
\text { POAG? }\end{array}$} & \multirow[b]{2}{*}{$O R$} & \multirow[b]{2}{*}{ Association IOP? } & \multirow[b]{2}{*}{$O R$} \\
\hline & & & & & Diabetes & $P O A G^{\star}$ & & & & \\
\hline Waite et al & 1935 & Massachusetts & clinic & 2002 & clinic & $1,2,3$ & no & NA & NA & NA \\
\hline Palomar & 1956 & Spain & clinic & 416 & clinic & 1 & no & NA & yes lower in DM & NA \\
\hline Armstrong et al & 1960 & Texas & clinic & 998 & clinic & $1,2,3$ & yes & 2.9 & i & 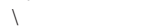 \\
\hline Sugar & 1964 & - & - & - & - & - & yes & NA & - & - \\
\hline Newell & 1965 & - & - & - & - & - & yes & NA & - & - \\
\hline Beckert† & 1966 & Washington & clinic & 304 & clinic & 1,2 & - & - & yes higher in DM & NA \\
\hline Safir et alt & 1966 & New York & clinic & 107 & clinic & 1 & - & - & yes higher in DM & NA \\
\hline Armaly and Baloglou & 1967 & Lebanon & clinic & 390 & clinic & 1 & - & - & yes lower in DM & NA \\
\hline Bankes & 1968 & Bedford & Community & 3072 & Urinalysis, GTT & $1,2,3$ & - & - & no difference & - \\
\hline Bouzas et al & 1971 & Boston & clinic & 101 & clinic & 1 & - & - & no difference & - \\
\hline Morgan and Drance & 1975 & Canada & Mixed & NS & Interview & $1,2,3$ & no & NA & - & - \\
\hline Leske and Podgor & 1983 & Framingham & Community & 2315 & GTT & 1,3 & no ${ }^{\star \star}$ & NA & yes higher in DM & 2.3 \\
\hline Klein et alt†† & 1984 & Winsconsin & Community & 2747 & clinic & 1 & yesł & 1.84 & yes higher in DM & NA \\
\hline David et al & 1987 & Israel & Community & 2547 & Interview & 1,2 & - & - & yes higher in DM & 1.58 \\
\hline Wilson et al & 1987 & Boston & clinic & 441 & Interview & 1,2 & yes & 1.6 & - & - \\
\hline Klein et al & 1994 & Beaver Dam & Community & 4926 & $\mathrm{HbA}_{1 \mathrm{c}}, \mathrm{RBG}$ & $1,2,3$ & yes & 1.68 & yes higher in DM & NA $\neq \neq$ \\
\hline Tielsch et al & 1994 & Baltimore & Community & 5308 & Interview & $1,2,3$ & no & 1.03 & yes higher in DM & $1.53^{\star \star \star}$ \\
\hline Dielmans et al & 1996 & Rotterdam & Community & 4012 & GTT & $1,2,3$ & yes & 3.11 & yes higher in DM & $0.31 \mathrm{~mm} \mathrm{Hg}$ \\
\hline
\end{tabular}

${ }^{\star}$ Criteria required for diagnosis of POAG: 1 (IOP), 2 (disc changes), 3 (field changes).

$\star \star$ Field defects not correlated with diabetes.

$\star \star \star$ Authors' calculation from published figures.

†Children and young type 1 diabetic patients included.

t+No non-diabetic controls.

††624 patients excluded because IOP measurement unreliable.

$\ddagger$ Reported glaucoma-not examined by authors.

$\$ \$ 8.5 \%$ Diabetic patients IOP $>21 \mathrm{~mm} \mathrm{Hg}$ cf $4.3 \%$ IOP $>21 \mathrm{~mm} \mathrm{Hg}$ controls.

was $1.1 \%$. Diabetes was present in 51 patients $(1.7 \%)$. Although the IOP was greater than $21 \mathrm{~mm} \mathrm{Hg}$ in two, none of the 51 patients had any of the diagnostic criteria necessary to support a diagnosis of POAG. This study concluded that IOPs in diabetic patients were no different from controls and found no evidence of an association between diabetes and POAG.

In a case-control, questionnaire study Wilson et $a l^{29}$ studied 83 confirmed and 121 possible glaucoma cases (total 204) selected from a hospital clinic. Control patients (237) were from the same ophthalmic clinics in whom a diagnosis of glaucoma had been excluded. A questionnaire established history of drug exposure, race, family history, and smoking habits. Diabetes, hypertension, and myopia were defined by interview. All variables were analysed by a multivariate regression model to define risk factors for POAG. A case of glaucoma was defined as IOP $>21 \mathrm{~mm}$ $\mathrm{Hg}$ if associated with large or asymmetric disc cupping. There were no requirements for a diagnostic field change. Cases were also defined as glaucomatous if IOP $>30 \mathrm{~mm}$ $\mathrm{Hg}$ in both eyes regardless of disc appearance, thus inevitably misclassifying some cases of ocular hypertension. The authors reported a weak association with diabetes (adjusted rate ratio 1.6). The number of patients with diabetes was small (10 cases, 16 controls), however, which limited the power of the study and the significance of the observed association with diabetes.

These early studies contribute little meaningful information principally because of limitations in study design. More recently, four population based studies have been published which merit detailed discussion.

\section{Recent studies}

All subjects in the Framingham Study ${ }^{30}$ were screened for POAG by applanation tonometry and funduscopy. Subjects with elevated IOP or suspicious discs underwent Goldmann perimetry. There were 2315 subjects with complete data; and diabetic status was defined as part of the Framingham Heart Study. There was a direct relation between blood glucose and IOP $(15.3 \%$ of diabetic patients had an IOP $>21 \mathrm{~mm} \mathrm{Hg}$ in at least one eye compared with $9.8 \%$ in at least one eye for the group as a whole-adjusted odds ratio 2.3), but not between diabetes and a clinical diagnosis of POAG. There were 54 confirmed cases of POAG (prevalence $2 \%$ ). In a second report from the same group ${ }^{31}$ which examined cardiovascular risk variables and visual field defects, diabetes was increased twofold in those with IOP $>21 \mathrm{~mm} \mathrm{Hg}$. In this study visual field defects did not correlate with diabetes.

A sample of 2990 diabetic patients (1210 type 1 diabetes, 1780 type 2 diabetes) and 381 controls from the community based validated group of diabetic patients $(n=10135)$ in the Wisconsin Study of Diabetic Retinopathy ${ }^{32}$ were examined with Goldmann applanation and found that diabetic patients had higher IOPs than controls. Disc and field examination were not performed, but a higher reported rate of glaucoma among diabetic patients was found on health questionnaire. The significance of this finding is limited by the inclusion of rubeotic glaucoma patients (23 in the group with diabetes, none among the controls) although the trend was still noted after exclusion of these patients. The relatively small size of the control group and once again the fact that patients with diabetes were more likely to have been under the care of an ophthalmologist and to have been screened for POAG limits the interpretation of this study. This may represent Berkson's bias once again rather than any true association. Furthermore, the ascertainment of glaucoma by questionnaire may have led to overdiagnosis as there may be a tendency for subjects with ocular hypertension receiving treatment to believe they have glaucoma.

In 1994 Klein et $\mathrm{al}^{33}$ evaluated the relation between type 2 diabetes and POAG in subjects aged 43-84 years. This population based study defined glaucoma as the presence of suspicious or definite field defects (Henson CFS2000 perimeter) graded independently by four specialists. Disc changes and pressures were also examined; an IOP $>22$ $\mathrm{mm} \mathrm{Hg}$ was considered diagnostic of glaucoma. Diabetes was defined either by a history of previously diagnosed and treated diabetes $(n=395)$ or by a glycosylated haemoglobin greater than two standard deviations above the age matched mean or a random blood glucose greater than $11.1 \mathrm{mmol} / \mathrm{l}(\mathrm{n}=50)$. The study found a twofold increase of both definite and probable glaucoma in diabetic subjects (33 of 426 combined) compared with non-diabetic subjects (172 of 4420). They also found twice as many subjects had IOP $>21 \mathrm{~mm} \mathrm{Hg}$ in at least one eye among diabetic patients as among non-diabetics. 
The Baltimore Eye Survey ${ }^{34}$ examined 5308 subjects aged over 40 for a diagnosis of glaucoma. Ocular examination included applanation tonometry, stereo fundus photography of the discs and Humphrey automated or Goldmann perimetry. The diagnosis of POAG required open angles, disc cupping, and rim thinning with characteristic field changes required to match disc rim thinning in the absence of any other pathology. Elevation of IOP was not necessary for a diagnosis of glaucoma. A second ophthalmologist independently reviewed all cases.

In contrast with the rigorous definition of POAG, the presence of diabetes was defined by a positive response to a question about treatment for, or a history of, diabetes diagnosed in the past. A prevalence of diabetes of $10.6 \%$ among whites and $17.2 \%$ among blacks was found. The authors comment, reasonably, that differential reporting of diabetes between subjects with and without glaucoma is unlikely. Non-differential misclassification, however, will usually bias estimates of the odds ratio towards the null value and may mask any true effect. ${ }^{35}$ The study found no association between diabetes and POAG (odds ratio 1.03) in those examined and diagnosed as having glaucoma for the first time, but patients with a prior diagnosis of POAG showed a positive association with diabetes (odds ratio 1.7) again suggesting that referral bias may be responsible for some of the positive associations reported in previous clinic based studies. It is interesting that this figure is almost identical to that found in the clinic based study by Wilson et al..$^{29}$

Finally in the Rotterdam Study, ${ }^{36} 4012$ subjects aged 55 or over received full ocular examination for glaucoma, and a non-fasting oral glucose tolerance test for diabetes. Diabetic patients on antidiabetic medication were not given an OGTT and were therefore excluded. Glaucoma was defined by Goldmann applanation tonometry, Humphrey automated fields, and disc photography after instillation of a mydriatic. A visual field defect (carefully defined in the article) matching discs and classic for glaucoma was required for the diagnosis. Gonioscopy was performed on all patients with glaucomatous field defects. The study identified 37 patients with POAG (no other forms of glaucoma were identified) and for over half of these (53\%) the diagnosis was new. The authors reported a threefold increase in the presence of high tension glaucoma (crude odds ratio 4.18; age, sex, and hypertension adjusted OR 3.11) in people with diabetes and a diabetic OGTT. The numbers with normal tension glaucoma were too small for separate statistical analysis.

\section{Interpretation of existing studies}

Overall interpretation of the data from the above studies is fraught with difficulty. The definition of glaucoma differed too widely to allow meaningful comparison between early studies, and discrepancies in population and diabetes definition make comparison between most of the later studies similarly problematic.

Despite the relative ease with which patients with diabetes and POAG can be recruited from clinic populations, data from clinic based studies must be viewed with caution because both diabetes and POAG are underdiagnosed conditions and selection bias is therefore inevitable.

Studies based on screening of the general population have attempted to overcome this problem. The definition of diabetes by questionnaire rather than examination ${ }^{34} 37$ may introduce error because of recall bias and underestimate prevalence because of the large subclinical component of the disease. Studies that have based their findings on special screening programmes which have included biochemical confirmation of diabetes are to some extent limited by the fact that the number of patients with POAG detected by mass screening are relatively small. ${ }^{28} 313336$ Nevertheless, because the prevalence of diabetes in these populations (aged over 40) in developed countries is much higher than the average for the population as a whole, ${ }^{27} 38$ these studies may be adequately powered to detect an association, although this is not always the case. ${ }^{29}$

In addition to the above discussion, it should be stated that some studies have potentially underestimated glaucoma incidence because they have concentrated on people with type 1 diabetes too young to have an appreciable risk of developing POAG. ${ }^{38}$

All the above studies are prevalence based case-control studies - we found no published incidence based studies. In general, case-control studies are subject to survival bias, misclassification, and recall bias. Studies based on totally unselected populations minimise selection bias and are the only way of gaining reliable information about chronic, insidious, largely underdiagnosed conditions such as POAG and diabetes. If the population is too small, however, this can introduce bias of its own. In light of recent advances in the genetic epidemiology of POAG the possibility of founder effects in geographically defined populations has to be considered: the generalisability of such findings is still not assured.

Total population studies in defined areas have been achieved in Wales $(91.9 \% \text { of population covered })^{39}$ and Sweden ${ }^{40}$ but neither study looked at the incidence of, or relation to, diabetes in the same population. For studies examining this issue the closest to achieving total population coverage has been the Beaver Dam study ${ }^{33}$ which succeeded in including $83 \%$ of the township of eligible age. The authors looked at differences between participating and non-participating individuals and found no significant difference. The Baltimore survey ${ }^{34}$ used stratified cluster sampling and successfully screened $74.7 \%$ of eligible participants. However, in contrast with the Beaver Dam study this study found racial and age differences between participants and non-participants which may introduce some bias.

\section{Conclusion}

Screening all diabetic patients for POAG at the time of annual screening for retinopathy is an attractive proposition provided a clear clinical benefit could be demonstrated. ${ }^{41}$ A screening test should ideally be relatively inexpensive, simple, and quick to perform and if possible be capable of being administered by a nonspecialist. IOP testing by Tonopen would meet these criteria but would be too imprecise as a sole screening test. Further limiting the value of tonometry in this context are those studies which have suggested elevation of IOP in diabetes independent of POAG risk. ${ }^{14} 16313442$ Field testing would increase specificity but is technically demanding for the patient, expensive and, as a sole means of detecting glaucoma Klein and colleagues found, had a positive predictive power of only $16 \% .^{43}$ It is readily apparent that clear evidence that diabetic patients are indeed at significant risk of POAG is needed before screening can be considered worthwhile.

Meta-analysis of available data is impossible and accordingly it is not yet possible to define the attributable risk of glaucoma due to diabetes. Our current knowledge is insufficient to state with conviction that diabetes is a risk factor for the development of POAG. This is not to say that such a risk does not exist; only that conclusive evidence for it does not. To the best of our knowledge, only two adequately performed studies have borne out an association between the two diseases, ${ }^{33}{ }^{36}$ and one equally well 
conducted study (the largest in the world literature) has failed to confirm any association apart from that explained by referral bias. ${ }^{34}$

Further information from a prospective, general population based, incidence study of all subjects in a community with full ascertainment of diabetes would be helpful. This presents its own difficulties. Studies which have defined the prevalence of diabetes in an entire population have yielded inconsistent prevalence rates principally because of the difficulty in full ascertainment of type 2 diabetes, in particular diet controlled diabetes. ${ }^{44}$ The most accurate UK data from Tayside, Scotland, indicate that the prevalence of diabetes is $1.94 \%$, but increases to $5.3 \%$ in those aged $>55$ years who are at greatest risk of POAG ${ }^{45}$ If at least $5.3 \%$ of the population aged $>55$ years are diabetic and the ratio of diagnosed to undiagnosed POAG is assumed to be $50: 50,{ }^{6}$ based on 1996 census data ${ }^{46}$ there may be as many as 12000 diabetic patients with undiagnosed POAG in the United Kingdom if diabetic patients are not at excess risk of developing the disease. If, however, diabetic patients are at increased risk of developing POAG applying the lowest and highest odds ratios quoted in the literature (1.03 and 3.11 respectively) would mean a further 300 to 31200 patients may have undiagnosed POAG.

The above calculation assumes that the ratio of diagnosed to undiagnosed disease matches that of the general population. This is almost certainly not the case. Increased contact with community and hospital based ophthalmic services is likely to alter this ratio significantly, and may in fact more than compensate for any increased risk. The recent Royal College of Ophthalmologists recommendations state that diabetic patients are at increased risk of POAG: based on our review of the world literature we believe that there is too much conflicting evidence to extrapolate that screening be recommended for a subgroup of diabetic patients whose exact size, and even possible existence, remains in doubt.

John Ellis is a recipient of a Wellcome Trust training fellowship in clinical epidemiology.

J D ELLIS C J MACEWEN

Department of Ophthalmology, Ninewells Hospital and Medical School, Dundee

A D MORRIS

Medicines Monitoring Unit, Diabetes Centre, and University Department of Medicine, Ninewells Hospital and Medical School, Dundee

Correspondence to: Dr John Ellis, University Department of Ophthalmology, Ninewells Hospital and Medical School, Dundee DDI 9SY.

1 Jay JL, Murdoch JR The rate of visual loss in untreated primary open angle glaucoma $\mathrm{Br}$ F Ophthalmol 1993;77:176-8.

2 Crick RP. The epidemiology of glaucoma: the extent of the problem. In Crick RP, Caldwell ADS, eds. Glaucoma: proceedings of an international symposium on glaucoma, Brussels. London: Royal Society of Medicine, Academic Press, 1980:3-11 (International congress and symposium series, No 21).

3 Bankes JLK, Perkins ES, Tsolakis S, et al. Bedford glaucoma survey. BMF 1968;1:791-6.

4 Grey RHB, Burns-Cox CJ, Hughes A. Blind and partial sighted registration in Avon. Br f Ophthalmol 1989;73:88-94.

5 Department of Health and Social Security. Blindness and partial sight in England 1969-1976. London: HMSO, 1979 (Reports on Public Health and Medical Subjects, no 129) in Tuck MW, Crick RP. Testing and referral for glaucoma. Health Trends 1989;21:131-4.

6 Tuck MW, Crick RP. Screening for glaucoma. Why is the disease underdetected? Drugs Aging 1997;10:1-9.

7 Sheldrick JH, Ng C, Austin DJ, et al. An analysis of referral routes and diagnostic accuracy in cases of suspected glaucoma. Ophthalmic Epidemiol 1994;1:31-8.
8 Crick RP, Tuck MW. How can we improve the detection of glaucoma? BMf 1995;310:546-7.

9 Crick RP. Early detection of glaucoma. BMF 1982;285:1063-4.

10 Royal College of Ophthalmologists. Guidelines for the management of ocular hypertension and primary open angle glaucoma. London: RCO,1997.

11 Scottish Intercollegiate Guidelines Network. The care of diabetic patients in Scotland - prevention of visual impairment. Pilot edition, 1996:11.

12 Jain IS, Luthra CL. Diabetic retinopathy: its relationship with intraocular pressure. Arch Ophthalmol 1967;78:198.

13 Mapstone R, Clark CV. Prevalence of diabetes in glaucoma, BM7 1985;291: 93-5.

14 Becker B. Diabetes mellitus and primary open-angle glaucoma The XXVII Edward Jackson memorial lecture. Am f Ophthalmol 1971;71:1-16.

15 Sugar HS. Modern ophthalmology. 4th ed. London: Butterworths, 1964: Chapter 6:554.

16 Safir A, Poulsen E, Klayman J. Elevated intraocular pressure in diabetic children Diabetes 1964;13:161.

17 Armstrong JR, Dailv RK, Dobson HL, et al. The incidence of glaucoma in diabetes mellitus: a comparison with the incidence of glaucoma in the general population. Am f Ophthalmol 1960;50:55-63.

18 Newell F. Ophthalmology: principles and concepts. St Louis: Mosby, 1964: Chapter 24:399.

19 Waite JH, Beetham WP. Visual mechanisms in diabetes mellitus; comparative study of 2002 diabetics, and 457 non-diabetics for control. N Engl f Med 1935;212:367-429.

20 Palomar Palomar A. Manifestaciones oftalmologicas de la diabetes mellitus. Arch Soc Oftal Hispanoam 1956;16:827-1067.

21 Bankes JLK. Ocular tension and diabetes mellitus. $\mathrm{Br} \mathcal{F}$ Ophthalmol 1967;51:557-61.

22 Armaly MF, Baloglu PJ. Diabetes mellitus and the eye: II. Intraocular pressure and aqueous outflow facility. Arch Ophthalmol 1967;77:493-502.

23 Bouzas AG, Gragoudas ES, Balodimos MC, et al. Intraocular pressure in diabetes. Arch Ophthalmol 1971:85:423-7.

24 Dickersin K, Min YI. NIH clinical trials and publication bias. On-line f Curr Clin Trials [serial online] 1993;28 Apr:Doc no 50.

25 Becker B, Bresnick G, Chevrette L, et al. Intraocular pressure and its response to topical corticosteroids in diabetes Arch Ophthalmol 1966;76: 477-83.

26 Saffir A, Paulsen EP, Klaymen J, et al. Ocular abnormalities in juvenile diabetics. Arch Ophthalmol 1966;76:557-62.

27 Samanta A, Burden AC, Fent B. Comparative prevalence of non-insulin dependent diabetes mellitus in asian and white Caucasian adults. Diabetes Res Clin Practice 1987;4:1-6.

28 Bankes JLK. Ocular tension and diabetes mellitus. $\mathrm{Br} \mathcal{F}$ Ophthalmol 1967;51:557-61

29 Wilson MR, Hertzmark E, Walker AM, et al. A case-control study of risk factors in open angle glaucoma. Arch Ophthalmol 1987;105:1066-71.

$30 \mathrm{Kahn} \mathrm{HA}$, Milton RC. Alternative definitions of open-angle glaucoma: effect on prevalence and associations in the Framingham Eye Study. Arch Ophthalmol 1980;98:2172-7.

31 Leske MC, Podgor MJ. Intraocular pressure, cardiovascular risk variables, and visual field defects. Am f Epidemiol 1983;118:280-7.

32 Klein BEK, Klein R, Moss SF. Intraocular pressure in diabetic persons Ophthalmology 1984;91:1356-60.

33 Klein BEK, Klein R, Jensen SC. Open-angle glaucoma and older-onset diabetes. The Beaver Dam Eye Study. Ophthalmology 1994;101:1173-7.

34 Tielsch JM, Katz J, Quigley HA, et al. Diabetes, intraocular pressure, and primary open angle glaucoma in the Baltimore eye survey. Ophthalmology 1995;102:44-53.

35 Dosemeci M, Wacholder S, Lubin JH. Does nondifferential misclassification of exposure always bias a true effect toward the null value? Am $\mathcal{F}$ Epidemiol 1990;132:746-8.

36 Dielmans I, de Jong PTVM, Stolk R, et al. Primary open angle glaucoma, intraocular pressure, and diabetes mellitus in the general population. $O p h$ thalmology 1996;103:1271-5.

37 Morgan RW, Drance SM. Chronic open angle glaucoma and ocular hypertension. Br F Ophthalmol 1975;59:211-15.

38 Nielsen NV. The prevalence of glaucoma and ocular hypertension in type I and 2 diabetes mellitus: an epidemiological study of diabetes mellitus on the island of Falster, Denmark. Acta Ophthalmol 1983:61:662-72.

39 Hollows FC, Graham PA. Intraocular pressure, glaucoma, and glaucoma suspects in a defined population. Br F Ophthalmol 1966;50:570-86.

40 Stromberg U. Ocular hypertension. Frequency, course and relation to other disorders occurring in glaucoma, as seen from mass survey of all inhabitants over forty years of age in a Swedish town. Acta Ophthalmol (Kbh) 1962; (suppl) 69:1-75.

41 Anonymous. The screening muddle. [Editorial; comment] Lancet 1998;351: 459

42 David R, Zangwill L, Stone D. Epidemiology of intraocular pressure in a population screened for glaucoma. Brf Ophthalmol 1987;71:766-71.

43 Klein BEK, Klein R, Sponsel WE, et al. Prevalence of glaucoma. The Beaver Dam Eye Study. Ophthalmology 1992;10:1499-504.

44 Keen H. Limitations and problems of diabetes classification from an epidemiological point of view. In: Vranic M, Hollenberg CH, Steiner G, eds. Comparison of type I and type II diabetes. New York: Plenum Press, 1985:3146.

45 Morris AD, Boyle DIR, MacAlpine R, et al. The Diabetes Research and Audit in Tayside Scotland (DARTS) Study: electronic record linkage to create a district diabetes register. BMF 1997;315:524-8.

46 Wisniewski D, ed. Annual abstract of statistics 1998. London: Office for National Statistics, no 134 (data), 1996:10. 